\title{
Outcome of Vision Impairment and Diabetes Insipidus in Suprasellar Region Germinoma
}

\author{
Satoshi Utsuki , Hidehiro Oka ${ }^{1}$, Yoshiteru Miyajima', Chihiro Kijima', Kiyotaka Fujii', Shinichi Kan ${ }^{2}$ \\ ${ }^{1}$ Department of Neurosurgery, Kitasato University School of Medicine, Sagamihara, Japan; ${ }^{2}$ Department of Radiology, Kitasato \\ University School of Medicine, Sagamihara, Japan. \\ Email: utsuki@med.kitasato-u.ac.jp
}

Received November $18^{\text {th }}$, 2010; revised March 28 ${ }^{\text {th }}, 2011$; accepted April $5^{\text {th }}, 2011$.

\begin{abstract}
Aim: Many cases of suprasellar region germinoma occurs in diabetes insipidus (DI), but a patient initially may come to the hospital for the chief complaint of visual impairment. The aim of this study is to determine the etiology of initial symptom presentation and the outcomes of visual impairment and DI in suprasellar region germinoma. Methods: We investigated eleven cases of single lesion suprasellar germinomas that were diagnosed and treated in our hospital. For each, a magnetic resonance imaging (MRI) was performed. Results: At the hospital visit, decreased visual acuity was found in 5/11 cases, while DI was found in all cases. The decreased visual acuity was improved in 4/5 cases by treatment, but DI improved in only 2/11 cases. In 10 cases, DI occurred earlier than visual impairment. As the initial symptom, visual impairment occurred earlier than DI in only one case and did not improve by treatment. In this case, a pituitary stalk of the normal thickness could be identified by MRI, and the optic nerve was swollen. In ten cases except this case, no significant enlargement of optic nerve was detected, and a swollen pituitary stalk was confirmed. Conclusions: In suprasellar germinomas, it is rare, but the optic nerve can significantly swell at onset, while the pituitary stalk may be intact. In such cases, visual impairment occurs earlier than DI, and visual impairment may not be improved.
\end{abstract}

Keywords: Diabetes Insipidus, Neurohypophyseal Germinoma, Optic Nerve Germinoma, Visual Function

\section{Introduction}

Suprasellar germinomas are termed neurohypophyseal germinomas because they grow to border the hypothalamus - pituitary stalk — pituitary posterior lobe (the neuronhypophysis) [1]. Therefore, most cases develop diabetes insipidus (DI). Visual impairment can be found because the suprasellar germinoma's anatomical position is near the optic nerve. In many cases, treatment improves visual impairment, but it is possible that visual impairment will not improve [2]. There is no previous report that investigated initial symptom and its function outcome in detail in suprasellar germinoma. The aim of this study is a detailed investigation of initial symptoms and outcomes of DI and visual impairment in suprasellar region germinoma in order to determine the reason for these differences.

\section{Experimental Procedure}

We studied eleven cases of continued suprasellar region germinoma that were diagnosed and treated in Kitasato University hospital from 1995 to 2009. All of these cases were a single lesion of the suprasellar region, and investigation of detailed magnetic resonance imaging (MRI) was possible. One case of multiple lesions, including the suprasellar region, and a lesion outside of the suprasellar region was excluded. We investigated the initial symptom, symptom at the hospital visit, main locus of the tumor by MRI and the outcome after treatment.

\section{Results}

These cases ranged in age from six to 45 (mean 15) years old with three males and eight females. Biopsy was performed in eight patients; the pathological diagnosis was germinoma. In the other patient, neurohypophyseal germinoma was suspected based on MRI findings. Eight patients were treated with radiotherapy of 34Gy to whole brain, with a boost to the primary sites of $16 \mathrm{~Gy}$, in 160 cGy fractions daily. And three patients were treated with a combination of radiotherapy (30 - $40 \mathrm{~Gy}$ ) to whole ventricular system in 200 cGy fractions daily and two courses of chemotherapy (dose of $20 \mathrm{mg} / \mathrm{m}^{2}$ cisplatin and dose of $80 \mathrm{mg} / \mathrm{m}^{2}$ etoposide). At a median follow-up of 
96 months (range, 12 - 170 months), all patients are alive without evidence of persistent or recurrent disease. In six cases, the optic nerve was easily identified because the tumor was limited to neurohypophysis (Figure 1). In five cases, the size of the tumor made it difficult to determine the relationship of the optic nerves to the pituitary stalk by MRI (Figure 2 left). After treatment, the thick pituitary stalk gradually narrowed with tumor reduction (Figure 2 middle), and the optic nerves were not hard to identify due to the pituitary stalk (Figure 2 right). It was concluded that a tumor occurred from neurohypophysis in these four cases. The pituitary stalk without enlargement (Figure 3 left) and thick optic nerves (Figure 3 right) were observed with MRI after the treatment in the one other case. In this case, MRI had already found thick optic nerves before treatment (Figure 4 left). Furthermore, the pituitary stalk without enlargement that was understood by a comparison with the MRI after the treatment was also observed with gadolinium enhancement before the treatment (Figure 4 right).

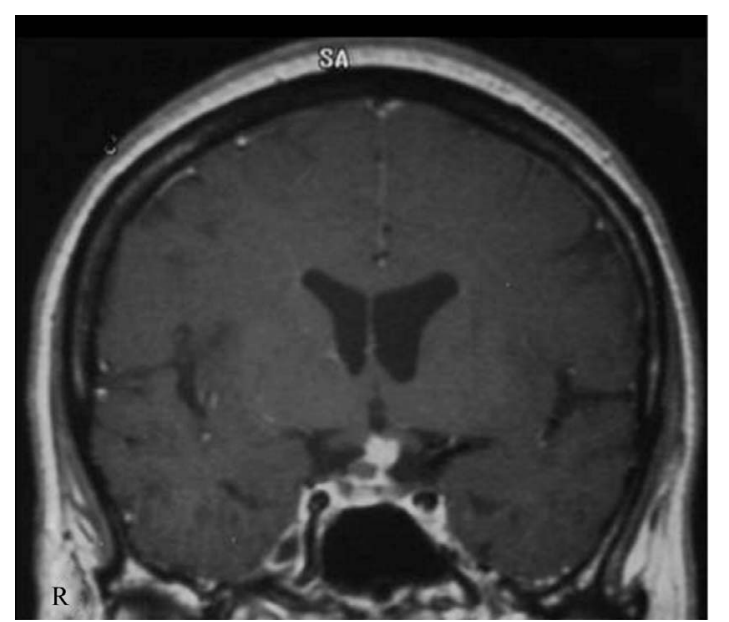

Figure 1. Coronal T1-weighted magnetic resonance image with enhancement of so-called nerurohypophyseal germinoma. The thick enhanced pituitary stalk and the optic nerves without enlargement can be identified easily, since the tumor is limited to nerurohypophysis.

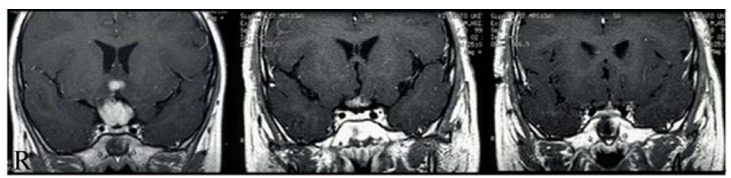

Figure 2. Pretreatment coronal T1-weighted magnetic resonance image with gadolinium enhancement showing a large lesion with homogenous enhancement in the suprasellar region (left). Because the tumor is large, the relation of optic nerve to the pituitary stalk cannot be identified. After treatment, the reduction of the tumor volume is seen, while the thick pituitary stalk with enhancement remains (middle). Optic nerves without enlargement are able to be identified above the enhanced pituitary stalk by MRI at the same time (right).

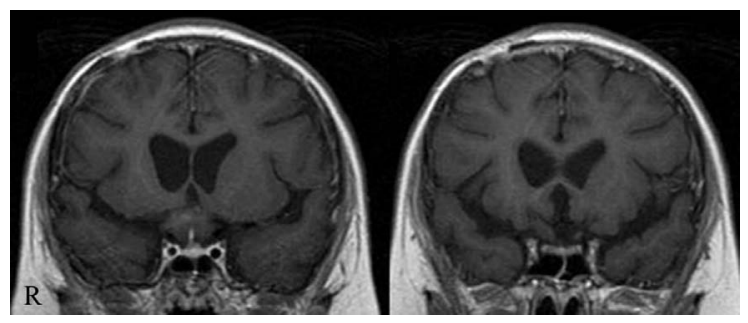

Figure 3. Posttreatment coronal T1-weighted magnetic resonance image with gadolinium enhancement of the same case as Figure 4. The intact homogenous enhanced pituitary stalk is can be observed (left). The mass lesion without the enhancement effect is seen above the pituitary stalk. The thick right optic nerve following this mass lesion is observed in the front of the region (right).

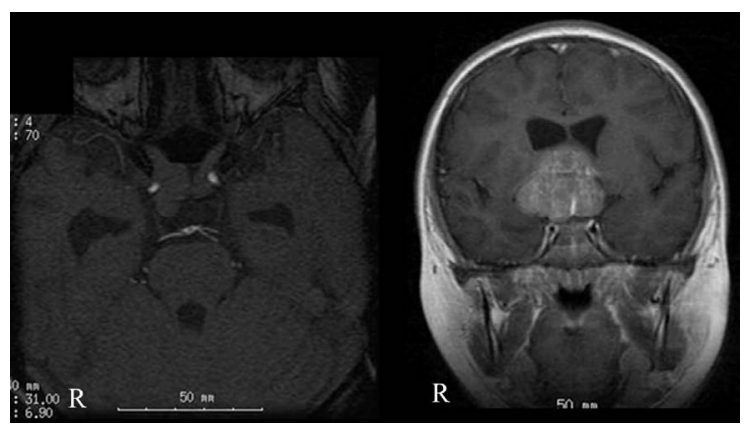

Figure 4. Pretreatment coronal T1-weighted magnetic resonance image (MRI). The thin slice MRI axial image shows the swelling of the right optic nerve (left). At postcontrast MRI, the suprasellar mass lesion had mild enhancement, and an enhanced linear structure at approximately center of the mass lesion can be seen (right). It was concluded that this was a pituitary stalk compared with Figure 3 left.

At the hospital visit, visual impairment and visual field disorders were found in five of 11 cases, and DI was found in all 11 cases. We reviewed in which cases DI preceded visual impairment and vice versa. DI preceded visual impairment in ten cases, whereas visual impairment preceded DI in only one case. DI was improved in 2 of 11 cases and the visual impairment was improved in 4 of 5 cases by treatment.

The case in which visual impairment preceded DI was also the case in which visual impairment continued. Enlargement of the optic nerve was observed by MRI at the hospital visit in this instance.

\section{Discussion}

Most intracranial germ cell tumors occur in the pineal body, the suprasellar region and the basal ganglia. Intracranial germ cell tumor rarely occurs in other regions $[3,4]$. Suprasellar germinomas are called neurohypophyseal germinomas because they grow in a line of the hypothalamic_pituitary stalk-neurohypophysis, i.e., the nerurohypophysis [1]. Optic nerve germinomas that oc- 
cur in the optic chiasm and the optic nerve are rarely reported [5-12] and differentiating between images of optic nerve germinoma and neurohypophyseal germinoma may be difficult when a tumor is large [8]. In other words, it may not be clear whether the site of the tumor origin is the optic chiasm and the optic nerve or the hypothalamus. In many cases, optic nerves with normal diameter and a swollen pituitary stalk can occur during tumor treatment. If this occurs while the tumor contracts, it can be unclear whether the tumor involves the optic nerve or is a new mass formation in front of the neurohypophseal germinoma without infiltrating the pituitary stalk. There is no difference in treatment according to the region of occurrence of the germinoma, but differentiation with craniophalyngioma, chiasmatic/hypothalamic low-grade gliomas, Langerhans cell histiocytosis and lymphocytic hypophysitis is needed when a tumor is large [13]. Also, it is necessary to differentiate optic nerve germinoma as well as optic sheath meningioma, infectious or granulomatous neuritis, orbital pseudotumor, lymphoma, medulloepithetlioma of the optic nerve and metastasis even if the mass is limited to the optic nerve [10]. Since a differential diagnosis of the cross tumor with imaging is limited, pathological diagnosis is required.

Suprasellar germinomas are often accompanied by DI with a reported incidence of $86 \%$ - 100\% [3,14-16]. DI was present in all cases in this study. Additionally, the frequency of visual impairment is reported as 50\% - 85\%; however, recent reports indicate that incidence is dropping with the assistance of newer imaging techniques $[2,3,14]$. It is reported that the improvement of the symptoms of DI with treatment is $50 \%$ or less, and improvement of visual impairment, including partial improvement, is $90 \%-100 \%[2,14]$. Germinomas can be accompanied by strong inflammation, and they are destructive lesions [17]. Improvement of symptoms is related to the degree of infiltration of the tumor cells. If the main locus of suprasellar germinoma is the neurohypophysis, and the tissue destruction is allowed to progress, hypopituitarism will develop. Suprasellar region germinoma tumor cells and inflammation can alternatively cause visual impairment since the neurohypophysis and the optic nerves are nearby anatomically. In many cases, the symptoms disappear by the decrement of the tumor since the symptoms were caused only by the compression of the tumor without infiltration. Also, visual impairment will be improved since infiltration and destruction of the optic nerves are minor, even if the visual impairment is caused by infiltration of the tumor and its inflammatory influence. However, visual impairment will not be improved if destruction of the optic nerve occurs by infiltration of many tumor cells and enlarges the optic nerve, as in this case $[6,10,11]$.
Suprasellar region germinoma often presents with DI as the initial symptom [18]. However, visual impairment may precede DI in suprasellar germinomas. No detailed cases of visual impairment preceding DI in suprasellar germinomas have been reported in the past. When a tumor is not limited to the optic nerve, as in optic nerve germinoma, visual impairment precedes DI [8]. This is because tumor cells infiltrate the optic nerve more so than the nerurohypophysis. In all neurohypophysis germinomas, infundibular thickening (up to $16 \mathrm{~mm}$ ) was usually observed [16]. An intact pituitary stalk and an optic nerve enlargement were observed for one case in which visual impairment preceded DI. This indicated that tumor cells were infiltrating the optic nerve more so than the pituitary stalk. These germinomas may have occurred in the optic nerve.

\section{Conclusions}

Phenomenological approach enables express-evaluation of the functional state of health for men with non-specific physicochemical parameters. Such as integral intensity of oscillator, effective ionization potential, electron affinity, parameters of identification [6].

\section{REFERENCES}

[1] I. Fujisawa, R. Asato, R. Okumura, Y. Nakano, T. Shibata, D. Hamanaka, T. Hashimoto and J. Konishi, "Magnetic Resonance Imaging of Neurohypophyseal Germinomas,” Cancer, Vol. 68, No. 5, 1991, pp. 10091014.

doi:10.1002/1097-0142(19910901)68:5<1009::AID-CNC R2820680517>3.0.CO;2-R

[2] N. Saeki, H. Murai, M. Kubota, N. Fujimoto and A. Yamaura, "Long-Term Karnofsky Performance Status and Neurological Outcome in Patients with Neurohypophyseal Germinomas,” British Journal of Neu- rosurgery, Vol. 15, No. 5, 2001, pp. 402-408. doi:10.1080/02688690120082404

[3] M. Matsutani, K. Sano, K. Takakura, T. Fujimaki, O. Nakamura, N. Funata and T. Seto, "Primary Intracranial Germ Cell Tumors: A Clinical Analysis of 153 Histologically Verified Cases," Journal of Neurosurgery, Vol. 86, No. 3, 1997, pp. 446-455. doi:10.3171/jns.1997.86.3.0446

[4] S. Utsuki, H. Oka, Y. Tanizaki, K. Kondo and K. Fujii. "Radiological Features of Germinoma Arising from Atypical Locations," Neurologia Medico-Chirurfica (Tokyo), Vol. 45 No. 5, 2005, pp. 268-271. doi:10.2176/nmc.45.268

[5] P. Perrini, L. Ventura, A. Ricci and R. Galzio, "Primary Germinoma of the Orbit,” Neurosurgery, Vol. 57, No. 4, 2005, pp. E813.

[6] P. Krolak-Salmon, G. Androdias, J. Honnorat, C. Caudie, P. Bret, D. Hernette and A. Vighetto, "Beware of Optic Neuritis,” Lancet Neurol, Vol. 1 No. 8, 2002, pp. 516-517. doi:10.1016/S1474-4422(02)00225-9 
[7] H. Iizuka, T. Nojima and S. Kadoya, "Germinoma of the Optic Nerve: Case Report,” Noshuyo Byori, Vol. 13, No. 2, 1996, pp. 95-98.

[8] J. T. Wilson, S. L. Wald, P. A. Aitken, J. Mastromateo and P. T. Vieco, "Primary Diffuse Chiasmatic Germinomas: Differentiation from Optic Chiasm Gliomas," Pediatric Neurosurgery, Vol. 23, No. 1, 1995, pp. 1-6. doi:10.1159/000120927

[9] S. Rath, G. K. Vemuganti, G. Biswas and H. Mod, "Optic Nerve and Chiasmal Germinoma,” Ophthalmic Plastic \& Reconstructive Surgery, Vol. 25, No. 2, 2009, pp. 161-163. doi:10.1097/IOP.0b013e31819aacbb

[10] M. L. DiLuna, A. M. Two, G. H. Levy, T. Patel, A. J. Huttner, C. C. Duncan and J. M. Piepmeier, "Primary, Non-Exophytic, Optic Nerve Germ Cell Tumors,” Jou nal of Neuro-Oncology, Vol. 95, No. 3, 2009, pp. 437-443. doi:10.1007/s11060-009-9941-1

[11] T. D. Nadkarni, S. C. Fattepurkar, K. I. Desai and A. Goel, "Intracranial Optic Nerve Germinoma,” Journal of Clinical Neuroscience, Vol. 11, No. 5, 2004, pp. 559-561. doi:10.1016/j.jocn.2004.02.006

[12] C. B. Bowman and B. K. Farris, "Primary Chiasmal Germinoma. A Case Report and Review of the Literature," Journal of Clinical Neuro-Ophthalmology, Vol. 10, No. 1, 1990, pp. 9-17.

[13] M. Warmuth-Metz, A. K. Gnekow, H. Muller and L. Solymosi, "Differential Diagnosis of Suprasellar Tumors in Children,” Klin Padiatr, Vol. 216, No. 6, 2004, pp. 323-330. doi:10.1055/s-2004-832358
[14] H. Oka, N. Kawano, T. Tanaka, S. Utsuki, I. Kobayashi, H. Maezawa and K. Fujii, "Long-Term Functional Outcome of Suprasellar Germinomas: Usefulness and Limitations of Radiotherapy,” Journal of Neuro-Oncology, Vol. 40, No. 2, 1998, pp. 185-190. doi:10.1023/A:1006184100834

[15] D. H. Nam, K. C. Wang, C. H. Shin, S. W. Yang and B. K. Cho, "A Simple Method of Predicting Hormonal Outcome in Children with Intracranial Germinoma," Child's Nervous System, Vol. 15, No. 4, 1999, pp. 179-184. doi:10.1007/s003810050365

[16] M. Kanagaki, Y. Miki, J. A. Takahashi, Y. Shibamoto, T. Takahashi, T. Ueba, N. Hashimoto and J. Konishi, "MRI and CT Findings of Neurohypophyseal Germinoma," European Journal of Radiology, Vol. 49, No. 3, 2004, pp. 204-211. doi:10.1016/S0720-048X(03)00172-4

[17] S. Utsuki, H. Oka, Y. Tanizaki, K. Kondo, N. Kawano and K. Fujii, "Pathological Features of Intracranial Germinomas with Reference to Fibrous Tissue and Granulomatous Change,” Brain Tumor Pathol, Vol. 22, No. 1, 2005, pp. 9-13. doi:10.1007/s10014-004-0171-0

[18] M. Bettendorf, M. Fehn, J. Grulich-Henn, B. Selle, K. Darge, D. K. Ludecke, U. E. Heinrich and W. Saeger, "Lymphocytic Hypophysitis with Central Diabetes Insipidus and Consequent Panhypopituitarism Preceding a Multifocal, Intracranial Germinoma in a Prepubertal Girl," European Journal of Pediatr, Vol. 158, No. 4, 1999, pp. 288-292. doi:10.1007/s004310051074 